\title{
Methodology for assessing measures to reduce the technological risk caused by the effects of electromagnetic factor at the enterprises of the fuel and energy complex of Russia
}

\author{
T.A. Alimkhanova, S.V. Kovshov \\ Saint-Petersburg Mining University, Saint Petersburg, Russia
}

\begin{abstract}
The study includes assessment of working conditions of personnel, modeling of non-ionizing radiation distribution, methods and means of protection contributing to the effective organization of measures to protect workers. The study analyzes the actual parameters of electromagnetic safety at the facility for transportation and storage of natural gas; a mechanism is proposed for modeling the electromagnetic effect of the transformer on an adjacent work area; the option of minimizing the negative impact of the transformer by shielding is proposed; the effectiveness parameters of the developed shield are estimated.
\end{abstract}

\section{Hygienic regulation of electromagnetic fields}

Electromagnetic fields (EMFs) are one of the sources that affect human health [1]. High biological activity was noticed by scientists in the early twentieth century. Research results were obtained using radar tools [2]. There are many sources of EMFs in everyday life. This leads to electromagnetic pollution of the environment [3]. The World Health Organization published data, which confirmed the impact of this type of pollution on the human environment. World-class specialists keep studying the problem of electromagnetic safety, because it remains relevant.

Currents and charges create an electromagnetic field in space, which is characterized by vectors of electric and magnetic strength. The Maxwell equation connects the spatial and temporal derivatives of the vectors [4]. The levels of exposure to electric and magnetic fields should be evaluated separately. For example, magnetic field meters IMP-5 are used (Fig. 1). The equipment is shown to confirm that measurements were made in accordance with regulations. 


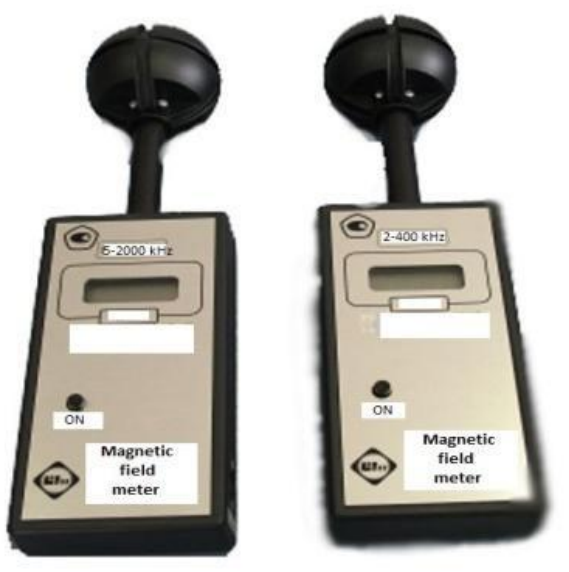

Fig. 1. Magnetic field meter.

\section{Measurement results}

The measurements were made in the power supply shop of the complex for liquefying natural gas. The electrician's workplace is located next to the automated workstation, next to the auxiliary needs panel and the direct-current board. The measurements results are presented in Tables 1 and 2 .

Table 1. Magnetic field results, NT

\begin{tabular}{|c|c|c|c|c|c|c|c|}
\hline Distance from sources & $\begin{array}{c}\text { Distance from } \\
\text { workers }\end{array}$ & & & & & & \\
\hline & 1.5 & 1 & 0.5 & 0 & 0.5 & 1 & 1.5 \\
\hline Height 0.5 m from the floor & & & & & & & \\
\hline 0.5 & 408 & 418 & 420 & 422 & 419 & 418 & 416 \\
\hline 0.75 & 341 & 345 & 352 & 357 & 352 & 354 & 356 \\
\hline 1 & 304 & 326 & 315 & 327 & 316 & 321 & 322 \\
\hline 1.25 & 250 & 254 & 271 & 276 & 278 & 269 & 243 \\
\hline 1,5 & 134 & 148 & 160 & 163 & 161 & 158 & 140 \\
\hline 1.75 & 31 & 35 & 53 & 55 & 50 & 48 & 37 \\
\hline Height 1 m from the floor & & & & & & & \\
\hline 0.5 & 517 & 524 & 530 & 535 & 531 & 520 & 519 \\
\hline 0.75 & 409 & 438 & 439 & 435 & 420 & 414 & 411 \\
\hline 1 & 446 & 450 & 468 & 470 & 469 & 445 & 440 \\
\hline 1.25 & 375 & 380 & 383 & 395 & 383 & 381 & 379 \\
\hline 1.5 & 207 & 225 & 237 & 255 & 346 & 245 & 236 \\
\hline 1.75 & 197 & 203 & 220 & 224 & 211 & 210 & 190 \\
\hline m from the floor & & & & & & & \\
\hline 0.5 & 641 & 655 & 680 & 698 & 678 & 654 & 640 \\
\hline 0.75 & 528 & 560 & 563 & 567 & 542 & 537 & 535 \\
\hline 1 & 399 & 405 & 432 & 437 & 433 & 426 & 417 \\
\hline 1.25 & 368 & 387 & 393 & 404 & 399 & 386 & 384 \\
\hline 1.5 & 245 & 249 & 261 & 268 & 256 & 250 & 234 \\
\hline 1.75 & 188 & 198 & 209 & 210 & 207 & 201 & 198 \\
\hline
\end{tabular}


Table 2. Electric field results, V / m

\begin{tabular}{|c|c|c|c|c|c|c|c|}
\hline Distance from sources & $\begin{array}{c}\text { Distance from } \\
\text { workers }\end{array}$ & & & & & & \\
\hline & 1.5 & 1 & 0.5 & 0 & 0.5 & 1 & 1.5 \\
\hline Height 0.5 m from the floor & & & & & & & \\
\hline 0.5 & 408 & 418 & 420 & 422 & 419 & 418 & 416 \\
\hline 0.75 & 341 & 345 & 352 & 357 & 352 & 354 & 356 \\
\hline 1 & 304 & 326 & 315 & 327 & 316 & 321 & 322 \\
\hline 1.25 & 250 & 254 & 271 & 276 & 278 & 269 & 243 \\
\hline 1,5 & 134 & 148 & 160 & 163 & 161 & 158 & 140 \\
\hline 1.75 & 31 & 35 & 53 & 55 & 50 & 48 & 37 \\
\hline from the floor & & & & & & & \\
\hline 0.5 & 517 & 524 & 530 & 535 & 531 & 520 & 519 \\
\hline 0.75 & 409 & 438 & 439 & 435 & 420 & 414 & 411 \\
\hline 1 & 446 & 450 & 468 & 470 & 469 & 445 & 440 \\
\hline 1.25 & 375 & 380 & 383 & 395 & 383 & 381 & 379 \\
\hline 1.5 & 207 & 225 & 237 & 255 & 346 & 245 & 236 \\
\hline 1.75 & 197 & 203 & 220 & 224 & 211 & 210 & 190 \\
\hline Height 1 m & & & & & & & \\
\hline 0.5 & 641 & 655 & 680 & 698 & 678 & 654 & 640 \\
\hline 0.75 & 528 & 560 & 563 & 567 & 542 & 537 & 535 \\
\hline 1 & 399 & 405 & 432 & 437 & 433 & 426 & 417 \\
\hline 1.25 & 368 & 387 & 393 & 404 & 399 & 386 & 384 \\
\hline 1.5 & 245 & 249 & 261 & 268 & 256 & 250 & 234 \\
\hline 1.75 & 188 & 198 & 209 & 210 & 207 & 201 & 198 \\
\hline
\end{tabular}

The obtained values must be compared with the maximum permissible level (MPL). All measurements were made in the frequency range $5 \mathrm{~Hz}-2 \mathrm{kHz}$. The average value of the magnetic flux density at a height of $0.5 \mathrm{~m}$ from the floor and at a distance of $0.5 \mathrm{~m}$ from the worker is $420 \mathrm{NT}$, which is 1.7 times higher than the MPL (250 NT). At a height of $1.0 \mathrm{~m}$ from the floor and at a distance of $0.5 \mathrm{~m}$ from the employee, it amounted to - 524 NT, which exceeds the MPL by 2.1 times, and at a height of $1.4 \mathrm{~m}$ from the floor and the same distance, to -663 NT, which exceeds the MPL by 2.7 times.

The average values of the electromagnetic field strength are within the normal range and do not exceed the MPL $(25 \mathrm{~V} / \mathrm{m})$.

In this mode of operation of the enterprise, the class of working conditions is defined as harmful 3.1. The harmful factor is the magnetic field in the frequency range of $5-2000 \mathrm{~Hz}$ (Order of the Ministry of labor and social protection of Russian Federation 2014).

\section{Modeling}

A method is needed to visualize the distribution of EMF from equipment for convenient presentation of measurements and studies. Presently, a lot of programs today are available (FEMM, Maxwell, MAGNET, etc.), but it is important to take into account the characteristics of radiating objects, climatic conditions and other factors. As you know, transformers and switchgears are sources of electromagnetic fields of industrial frequency $(50 \mathrm{~Hz})$. This study uses a program for simulating EMF from these devices, namely, EFC-400 (Magnetic and Electric Field Calculation). Firstly, approximate location boundaries were built. This helped create the factory conditions at. Secondly, models of the transformer and the open switchgear (Fig. 2) were implemented. 


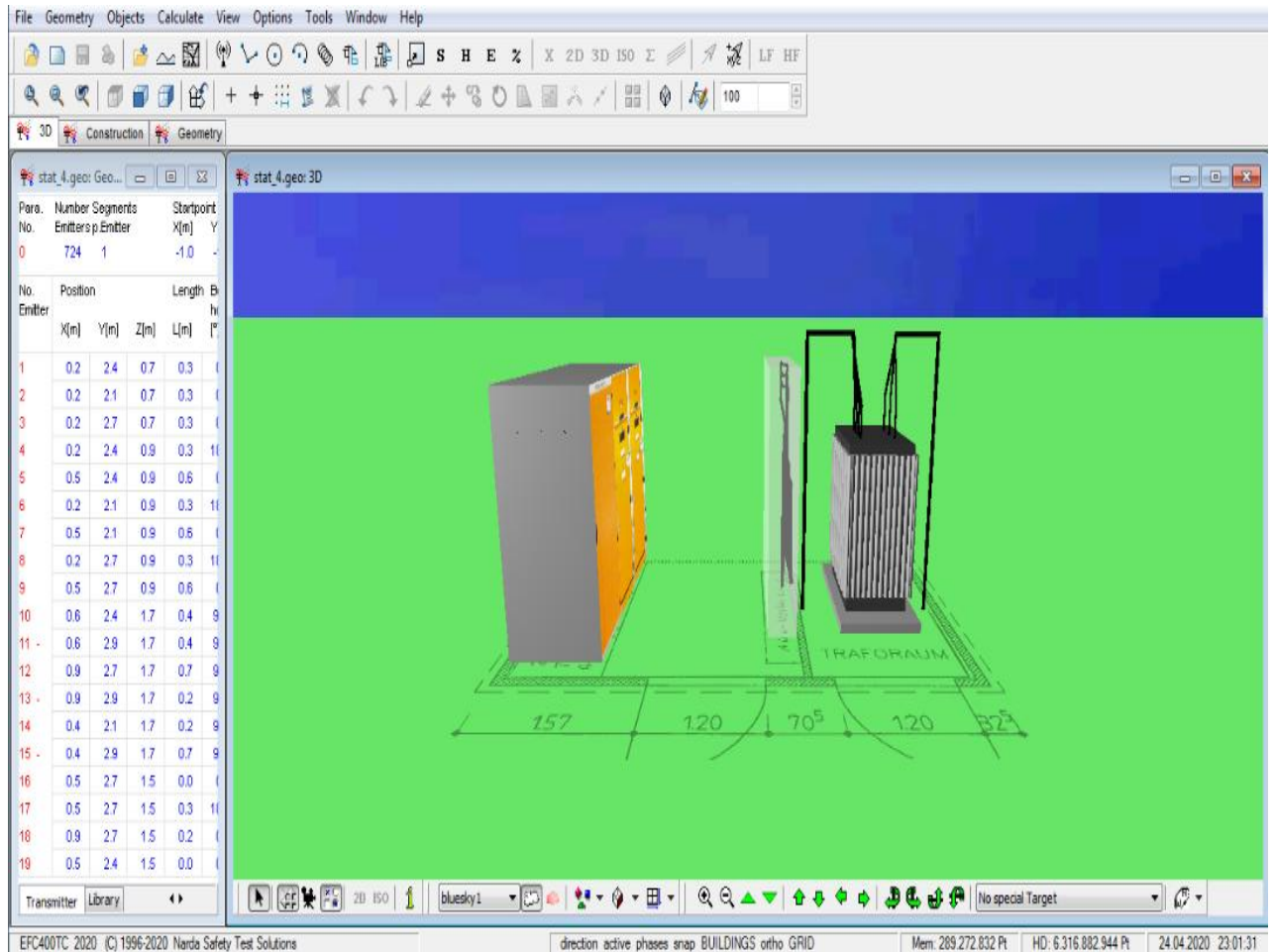

Fig. 2. Models of outdoor switchgear and transformer

The next step was the introduction of the characteristics of these devices and the construction of a magnetic field at given points in space. The levels of the acting fields are displayed on the map (Fig. 3).

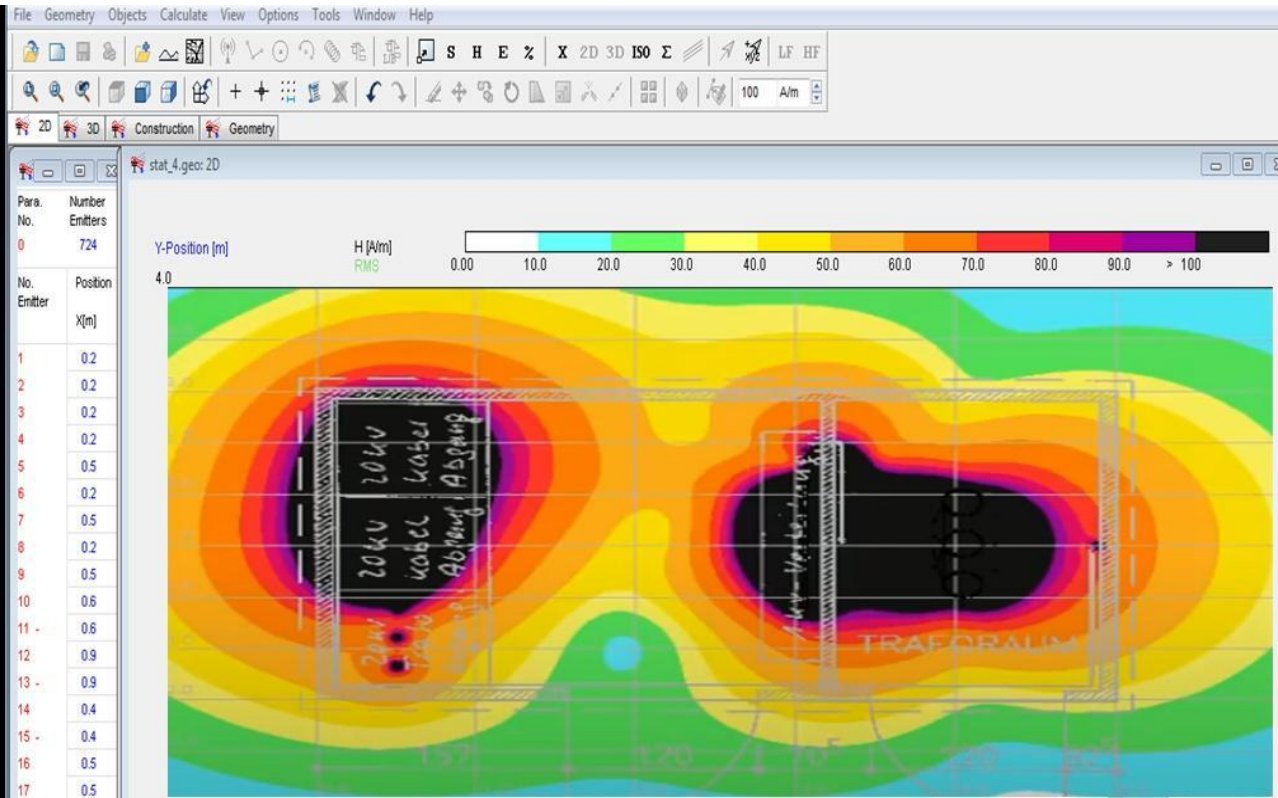

Fig. 3. Magnetic field 
Based on the indicators of the program we can conclude that the electrical equipment does not affect the magnetic field above the maximum allowed level [5]. But in conjunction with other sources of radiation and the time that personnel spend in this area, these fields overlap and their combined level of exposure increases significantly.

\section{Developments of protective measures}

\subsection{Shielding}

An electromagnetic shield is a design to weaken the effects of electromagnetic fields. The main characteristic of the protective screen is the shielding efficiency, which can be calculated using the following formulas [6]:

$$
\begin{gathered}
\ni=\frac{E}{E_{\ni}}, \\
Э=\frac{H}{H_{\ni}}, \\
Э=\frac{S}{S_{\ni}},
\end{gathered}
$$

Where $\mathrm{E}_{\ni}, \mathrm{H}_{\ni}, \mathrm{S}_{\ni}=$ electric and magnetic field strengths with a shield; E,H,S = electric and magnetic field strengths without shield.

The shielding efficiency is expressed in $\mathrm{dB}$. It is important to take into account geometric dimensions and the material of which the shield is made. Shielding can be implemented by some structures [4]:- premises, cameras, structures. They are presented by stationary and mobile variations. Steel sheets 2-3 mm thick can absorb EMF 60-120 dB. These structures are equipped with doorways, gates, filters, ventilation systems, fire extinguishing systems, etc., which ensure normal operation. Shielding materials are metal sheets and nets. A solid sheet provides the best protective function. The effect can reach 100 or more $\mathrm{dB}$. Creating a seam is one of the determining factors when choosing a sheet thickness. However, steel sheets are more expensive. Manufacturing, repairs and maintenance require more attention and more costs. Many factors affect the reliability and durability of these elements. This requires the elimination of defects.

Metal mesh shields with brass or tinned steel wire from 0.25 to $6 \mathrm{~mm}$ are less effective. The reflection of an electromagnetic wave from the surface of the mesh is the main defining property of shielding. The effect when using a screen made of a tinned low-carbon mesh reaches 50-60 dB, and from a double mesh with a layer spacing of $100 \mathrm{~mm}-90 \mathrm{~dB}$.Also, foil materials, conductive paints, and adhesives are used.

The most effective are mesh shields, which have the greatest economic efficiency.

It is important to take into account some difficulties connected with changes in the design and production technology. Therefore, it is recommended to install shields not around a specific installation, but on those parts of the equipment that emit the largest EMFs. It is also necessary to carefully select the joints of these structures, - in order to prevent the spread of the electromagnetic field through the seams. It was decided to use a screen with the following parameters: size $2 \times 4 \mathrm{~m}$, steel wire $0.21 \mathrm{~mm}$ thick, mesh size 0.3 $\times 0.3 \mathrm{~mm}$. The step from the radiation source is $0.2 \mathrm{~m}$.

The required efficiency of the shielding device [7] is:

$$
\Delta_{\text {required }}=20 \lg \frac{H}{H_{\text {norm }}}=20 \lg \frac{1980}{250} 20 \lg 7,92=17,98 \mathrm{~dB} .
$$

The equivalent shield radius is: 


$$
R=\sqrt[3]{\frac{3 V}{4 \pi}}=\sqrt[3]{\frac{3 * 2 * 4 * 0,2}{4 * 3,14}}=0,7 \mathrm{~m}
$$

Where $V=$ volume.

The shielding parameter is:

$$
\gamma=\frac{d}{2 \pi R}\left(\ln \frac{d}{r}-1,25\right)=\frac{0,3}{2 * 3,14 * 0,7}\left(\ln \frac{0,3}{0,105}-1,25\right)=4,7 * 10^{-3},
$$

where $d=$ cell pitch; $r=$ grid radius.

Permeability of the screen is al follows:

$$
\mu=\frac{3 \gamma}{1+3 \gamma}=\frac{3 * 4,7 * 10^{-3}}{1+3 * 4,7 * 10^{-3}}=13,9 * 10^{-3} .
$$

The actual screen efficiency is:

$$
\Delta_{\text {actual }}=20 \lg \lg \left(\frac{1}{\mu}\right)=20 * \lg \lg \left(\frac{1}{13,9 * 10^{-3}}\right)=37,1 \mathrm{~dB} .
$$

Verification of conditions: $17.98 \mathrm{~dB}<37.1 \mathrm{~dB}$. The condition is satisfied.

The proposed protective screen will bring down the class of working conditions to permissible.

\subsection{Shielding plaster}

Every year, research in the field of studying methods of protection against non-ionizing radiation becomes more widespread [8]. There are other means for shielding, for example, conductive paints, plasters, etc $[9,10,11]$. These materials are convenient for applying onto walls. Compared to the mesh shielding of individual sections, applying to the surface of the plaster is much simpler. With the right composition, it can be also frost-resistant,-; to obtain the required efficiency, one layer of $1 \mathrm{~mm}$ is enough.

The disadvantages include the need for grounding and topcoating of some primers. The color should be black. Not all materials are frost-resistant, which is a particularly important property for shielding the premises of the fuel and energy complex in the natural conditions of Russia. As conducting materials, carbon black, graphite, etc. are used.

\subsection{Personal protective equipment (PPE)}

EMF clothes include suits, gowns, raincoats, helmets, goggles, etc. Such products are made of reflective or absorbing materials (steel, copper, metal oxides, tin), by spraying metals.

Currently, a wide variety of materials, fabrics, sets have found broad application in the creation of personal protective equipment against electromagnetic fields. And these products are quite popular. The use of suits to protect the personnel of the power supply shop contributes to the positive effect. But when choosing between other means with equal effectiveness, it is necessary to take into account both technological and economic parameters. For example, the cost of purchasing PPE will be higher than shielding. It is necessary to consider the number of people in the workshop.

\section{Conclusion}

The following results were obtained:

- Hygienic assessment of the levels of exposure to the electromagnetic field on workers

- In the EFC-400 program, models of a transformer and open switchgear were constructed and the distribution of the magnetic field was modeled 
- Protective measures have been proposed.

As a result, the objectives of the study were achieved:- with the help of software modeling, the effect of electromagnetic radiation on employees of the power supply shop was fully determined suitable protective measures were proposed, and their effectiveness was tested, the prerequisites were created for studying the properties of certain shielding materials in laboratory conditions.

The disadvantages of the proposed solution include complex structural changes at the enterprise. Therefore, additional methods are suggested. This is the creation of a shielding solution and the application of shielding plaster to the radiating equipment. The advantages of this tool include economic benefits compared to other methods.

Employees and employers are advised to reduce the time spent in the work area near radiation installations.

\section{References}

1. V. G. Eremin, V. V. Safronov, A. G. Skhirtladze, , G. A Kharlamov., ed., Safety in the energy sector: textbook for students of higher educational institutions. Moscow: Academy publishing (2010).

2. D. H. Shapiro, ed. Fundamentals of the theory of electromagnetic shielding. L: Energiya (1975).

3. G. V. Fedorovich, ed., Ecological monitoring of electromagnetic fields. M. (2004).

4. S. G. Gendler, ed. Life safety: Hygienic assessment of working conditions.

5. I..A. Stepanyuk., Electromagnetic fields in geophysical processes and their influence on biological objects 154, 29 (2003).

6. N. I. Ivanov, ed., Safety of technological processes and productions. Moscow: Logo (2016).

7. M. L Rudakov, ed.Electromagnetic safety in industry. St. Petersburg: Polytechnic (1999).

8. Z. N. Cherkay, E. B.Gridina, ed., Life safety. Physiology and occupational health. Saint-Petersburg: Lema (2016).

9. Safety in the use of radiofrequency dielectric heaters and sealers. Geneva: International Commissions on Non-Ionizing Radiation Protection (1998).

10. S.V. Kovshov, T.A. Alimkhanova, V.M. Kitsis Ranking of the section of East Siberia Pacific Ocean main pipeline by accident probability using bow-tie method and CheckList support tool11(3), 1-18 (2020).

11. S.V. Kovshov, T.A. Alimkhanova, V.M. Kitsis, Methodology for assessing the risk of emergencies the East Siberia - Pacific Ocean oil and gas main pipeline 1, 13-20 (2020). 